\title{
VALIDAÇÃO DA ESCALA DE ATITUDES DE PROFESSORES DE ESTATÍSTICA EM RELAÇÃO À ESTATÍSTICA NO ENSINO SUPERIOR NO BRASIL
}

\author{
The validation of a statistics teachers' attitude scale \\ in relation to the university statistcs teaching in Brazil
}

\author{
Ailton Paulo de Oliveira Júnior ${ }^{1}$ \\ José Fausto de Morais $^{2}$
}

\begin{abstract}
Resumo: O presente estudo destinou-se à construção e validação de uma escala de atitudes de professores de Estatística em relação à Estatística em cursos de Graduação no Brasil. A validação da Escala de Atitudes de professores de Estatística em relação à Estatística (EAPE) contou com uma amostra de 87 professores de sete estados brasileiros (Distrito Federal, Rio Grande do Sul, Paraná, Santa Catarina, São Paulo, Rio de Janeiro e Minas Gerais), de instituições de Ensino Superior públicas e privadas. Foi realizada análise fatorial experimental para a verificação da dimensionalidade da EAPE, sendo a solução de quatro fatores considerada a mais adequada. Os fatores extraídos foram denominados cognitivo, educativo, afetivo/social e de conduta, com alfas de Cronbach de 0,65; 0,68; 0,51 e 0,63 , respectivamente. A escala geral apresentou uma consistência interna de 0,76 .
\end{abstract}

Palavras-chave: Validade. Atitude. Estatística.

\begin{abstract}
This study aimed to build and validate a scale of attitudes of teachers of Statistics towards Statistics in the Graduate courses in Brazil. The validation of the scale of attitudes of teachers of Statistics in relation to Statistics (EAPE) had a sample of 87 teachers from seven Brazilian states (Distrito Federal, Rio Grande do Sul, Paraná, Santa Catarina, São Paulo, Rio de Janeiro and Minas Gerais) from institutions of higher education both public and private. Factor analysis was performed for the experimental verification of dimensionality of EAPE, andfour factors were considered most appropriate. The factors taken were called cognitive, educational, emotional/social and conduct, with the Cronbach alphas of $0.65,0.68,0.51$ and 0.63 , respectively. The scale showed a general internal consistency of 0.76 .
\end{abstract}

Keywords: Validity. Attitude. Statistic.

${ }^{1}$ Bacharel em Estatística, Doutor em Educação; Docente, Curso de Licenciatura em Matemática, Universidade Federal do Triângulo Mineiro. Uberaba, MG, Brasil. ailtonpaulo@matematica.uftm.edu.br

${ }^{2}$ Licenciado em Matemática, Mestre em Estatística Aplicada; Docente, Faculdade de Matemática, Universidade Federal de Uberlândia. Uberlândia, MG, Brasil.jfmorais@ffamat.ufu.br

${ }^{1}$ Rua Duque de Caxias, 250, apto. 3003

Uberaba, MG

$38.022-180$ 
Oliveira Júnior, A. P.; Morais, J. F.

\section{Introdução}

As atitudes dos professores afetam o ensino e os alunos, pois essas influenciam tanto as expectativas deles em relação aos alunos quanto o comportamento em relação aos mesmos. E essas ainda criam expectativas e comportamentos que influenciarão a autoimagem e o desempenho dos alunos, como observado por MacNab e Payne (2003).

Se, a princípio, os estudos sobre atitudes foram predominantemente focados em construtos unidimensionais, atualmente, os estudos multidimensionais são mais frequentes. Assim, os trabalhos de Auzmendi (1992), Gil Flores (1999), Gómez Chacón (2003) e Estrada, Batanero e Fortuny (2003) diferenciam as atitudes em três fatores básicos (afetivo, cognitivo e de conduta), também chamados de "componentes pedagógicos".

Estrada, Batanero e Fortuny (2003) distinguem dois tipos de componentes nas atitudes: componentes pedagógicos e antropológicos. Entre os primeiros mencionam: a) componente cognitivo, que são expressões do pensamento; b) componente afetivo, que são expressões de sentimento, e c) componente de conduta, que são componentes vinculados às atuações em relação ao objeto das atitudes Por outro lado, os componentes antropológicos são: a) componente social, que são as atitudes relacionadas com a percepção e valorização do papel da Estatística no âmbito sociocultural; b) componente educativo, que é o interesse em relação à Estatística e sua aprendizagem, e c) componente instrumental, que é a utilidade da Estatística em relação às outras disciplinas como forma de raciocínio e como componente cultural.

Desta forma, Onwuegbuzie (1998) utiliza um modelo multivariado para a predição do rendimento nas disciplinas de Estatística. Dedica-se fundamentalmente ao estudo da ansiedade e das atitudes dos professores, medidas por meio da escala de atitudes (ATS), desenvolvida por Gil Flores (1999). Entre suas conclusões, destacamos as correlações significativas entre o número de disciplinas de Estatística cursadas anteriormente ao momento da docência em Estatística e a pontuação obtida na escala de Atitudes ATS. Ao aplicar-se o modelo, Onwuegbuzie (2003), comprova que as atitudes e a ansiedade em relação à Estatística influem no resultado do processo ensino-aprendizagem, pois há uma motivação destes professores para criar uma aprendizagem significativa em suas classes para que seus alunos possam explorar diferentes metodologias e adquirirem segurança na sua própria capacidade, tanto os alunos quanto eles mesmos, os professores, e ainda valorizam o importante papel que tem esta disciplina na sociedade atual.

Nasser (1999) e Wisenbaker, Nasser e Scott (1999) realizaram vários estudos que analisam a relação entre as atitudes ou a ansiedade e o rendimento. Em Nasser (2004) é construído um modelo estatístico para predizer as atitudes de futuros professores em função de diferentes variáveis. Para isto analisa a possível relação entre as atitudes e a ansiedade em relação à Matemática e à Estatística, à aptidão matemática, à motivação e os resultados em Estatística de 167 professores em formação de língua árabe matriculados em cursos de introdução à Estatística em Israel. Dentre suas conclusões, indica que a aptidão matemática, a motivação, as atitudes em relação à Matemática e à Estatística e a ansiedade em relação às matemáticas explicam 36\% da variação do rendimento em Estatística.

Aparício, Bazán e Abdounur (2004) apresentam os resultados da aplicação de duas escalas de atitude em relação à Estatística (CAZORLA et al., 1999; ESTRADA, BATANERO e FORTUNY, 2003) e seu relacionamento com o desempenho na disciplina de Estatística em 
professores de Ensino Fundamental. O estudo foi feito com professores que participaram de um programa de complementação acadêmica à distância para obter o título universitário, e avaliado considerando um planejamento do tipo pré-teste e pós-teste durante a etapa presencial da disciplina. Os autores mostram que há uma mudança significativa e favorável na atitude em relação à Estatística medida nas duas escalas, e um relacionamento significativo desta atitude final com o desempenho na disciplina.

No Brasil o único trabalho encontrado por nós de validação para estudo em Educação Estatística é o de Cazorla et al. (1999), que validaram uma escala de atitudes para alunos de Estatística, adaptada a partir de outra adaptação e validação de uma escala de atitudes em relação à Matemática, de Brito (1998), considerando que esta escala se limita a aspectos afetivos, apresenta alta consistência interna, estabilidade no coeficiente de confiabilidade, validade de construto e de conteúdo, e levando em conta que as suas proposições são pertinentes à Estatística.

Há que se ressaltar a importância que a construção de escalas de atitudes podem assumir num contexto educacional quando se pretende aferir aspectos subjetivos do comportamento humano. Neste sentido, o emprego destes instrumentos torna viável a "transformação" de sentimentos, crenças e comportamentos, por exemplo, em dados numéricos, fornecendo, com isso, parâmetros de medida importantes. Estas medidas são particularmente interessantes em contextos de formação profissional e acadêmica na área da docência em Estatística, onde o preparo específico para a atuação em cenários que envolvem o ser humano em situações peculiares frequentemente demanda o domínio de atitudes claras e inequivocamente positivas frente às questões configuradas.

Uma validação consiste em avaliar se o instrumento mede aquilo que se propõe a medir e tem, como intenção, nortear a construção do instrumento quanto à forma de apresentação e conteúdo e sugestões quanto à retirada, acréscimo ou modificações dos itens que compõem o instrumento. Portanto, buscamos elaborar um instrumento válido e confiável que permitisse diagnosticar as atitudes dos professores de Estatística de Graduação em relação à Estatística.

Assim, o objetivo deste artigo é descrever as principais etapas da elaboração desta escala para avaliação de atitudes, bem como divulgar a versão final do instrumento construído.

\section{Método}

\section{Elaboração dos itens da Escala de Atitudes}

Os itens da escala de atitudes dos professores de Estatística em relação à Estatística (EAPE) foram elaborados com base na análise da literatura sobre atitudes de professores, a nossa experiência de mais de 13 anos como professor de Estatística e, ainda, nas trocas de experiência com colegas professores de Estatística.

Inicialmente, a escala EAPE, do tipo Likert, contava com 42 itens de proposições positivas e negativas - que expressam as atitudes de cada professor de Estatística em relação à Estatística.

Os 42 itens considerados inicialmente são os seguintes: (1) É divertido lecionar Estatística; (2) Motivar os alunos ajuda na aprendizagem da Estatística; (3) O pensamento estatís- 
tico é tão necessário para a cidadania eficiente como saber ler e escrever; (4) Respondo com maturidade quando meus alunos fazem perguntas; (5) Conscientizar os alunos quanto à importância da Estatística para suas atividades profissionais é perda de tempo; (6) Evito as informações estatísticas quando as leio; (7) Evito ler artigos científicos onde são apresentados resultados estatísticos; (8) Fico frustrado ao ensinar Estatística; (9) Gosto da Estatística porque ela ajuda a solucionar problemas objetivamente; (10) Utilizo muitas estatísticas sempre que desejo inspirar confiança; (11) Sinto-me frustrado com a incerteza dos modelos estatísticos; (12) Utilizo pouco a Estatística quando não estou em sala de aula; (13) Vejo de maneira incômoda as informações estatísticas apresentadas na mídia em geral; (14) O conhecimento de Estatística é como o de uma língua estrangeira: ele poderá ser útil a qualquer momento; (15) A aprendizagem da Estatística não pressupõe conhecimentos matemáticos; (16) As representações gráficas não facilitam a compreensão dos resultados estatísticos; (17) É importante apresentar conceitos básicos da Estatística Bayesiana; (18) A Estatística não é um instrumento de pesquisa confiável; (19) Comentar um problema de Estatística com colegas não ajuda a resolvê-lo; (20) Desenvolvo atividades utilizando probabilidades subjetivas em minhas aulas; (21) Vinculo a Estatística aos métodos e técnicas científicas; (22) Procuro diferentes maneiras de resolver um problema de Estatística; (23) É importante desenvolver pesquisas para que os alunos possam fazer relações entre a teoria e a prática; (24) A Estatística me ajuda a entender mais profundamente a complexidade de certos temas; (25) Para ser um bom professor de Estatística não é importante resgatar os conceitos estatísticos fundamentais; (26) Uma resposta aproximada da resposta certa é mais valiosa do que uma resposta certa de um problema aproximado; (27) Não me parece importante relacionar novos conceitos com conteúdos anteriormente apreendidos; (28) Os alunos devem estar conscientes da importância do conhecimento matemático para a aprendizagem da Estatística; (29) É importante apresentar as várias fórmulas para o cálculo do tamanho da amostra; (30) Podemos manipular a realidade por meio da Estatística; (31) Ao corrigir uma questão de Estatística concentro-me somente na precisão da resposta; (32) Ao corrigir uma avaliação de Estatística preocupo-me com a qualidade das argumentações apresentadas; (33) Devo desenvolver atividades com dados reais utilizando minhas experiências; (34) Vinculo os conteúdos estatísticos a minha experiência; (35) Estudo e procuro explicações lógicas que comprovem as imprecisões apresentadas por autores de livros de Estatística; (36) Ministro os conteúdos estatísticos de forma semelhante ao que faziam meus antigos professores; (37) Não desenvolvo atividades com dados reais utilizando experiências de outros; (38) Utilizo a Estatística exclusivamente para dar aulas; (39) Procuro evitar que os alunos memorizem os conceitos estatísticos; (40) Fazer perguntas aos alunos durante as aulas ajuda na apreensão do conteúdo; (41) Não julgo ser importante o conhecimento de softwares estatísticos por parte dos alunos; (42) $O$ plano de ensino não deve ser modificado durante o processo ensino-aprendizagem.

Para procedermos a estudos posteriores, cada uma das proposições positivas recebeu pontuação distribuída da seguinte forma: concordo totalmente $=5$ pontos; concordo parcialmente $=4$ pontos; nem de acordo, nem em desacordo (indiferença) $=3$ pontos; discordo parcialmente $=2$ pontos; e discordo totalmente $=1$ ponto. Para as negativas a pontuação foi: concordo totalmente $=1$ ponto; concordo parcialmente $=2$ pontos; nem de acordo, nem em desacordo (indiferença) $=3$ pontos; discordo parcialmente $=4$ pontos; e discordo totalmente $=5$ pontos. 


\section{Coleta de dados}

Os sujeitos de nossa amostra são 87 professores de Estatística de instituições de Ensino Superior públicas e privadas, que ministram disciplinas de Estatística em cursos que tenham esta importante ferramenta como conteúdo para a formação de seus profissionais no $1^{\circ}$ semestre letivo de 2008. Foram coletados os dados nos seguintes estados da Federação: Distrito Federal, Rio Grande do Sul, Paraná, Santa Catarina, São Paulo, Rio de Janeiro e Minas Gerais.

Para a coleta de dados, optou-se pela adoção de instrumento aplicado via Web, pelos problemas de acesso aos sujeitos da pesquisa e para assegurar a aleatoriedade do processo de seleção da amostra.

Para a análise foi utilizado o Microsoft Excel para a montagem do banco de dados, e, para a validação do instrumento, o pacote estatístico SPSS (Statistical Package for Social Science), uma poderosa ferramenta informática que permite realizar cálculos estatísticos complexos e visualizar, em poucos segundos, os resultados.

\section{Análise de dados}

A Análise Fatorial é uma técnica estatística, do conjunto de técnicas multivariadas, que serve aos propósitos de análise exploratória de um conjunto, com o intuito de reduzir um certo número de variáveis a uma dimensão menor, representado-as por meio de uma nova variável estatística que expressa uma combinação linear das variáveis originais, todas métricas ou quantitativas.

No estágio do processo de análise, consistiu na aplicação da Análise Fatorial Exploratória para a verificação da dimensionalidade das escalas. Os primeiros indicadores submetidos a essa análise foram as condições para a formação da intenção estratégica. Uma verificação do $\mathrm{KMO}=0,544$ e do Teste de Esfericidade de Bartlett $=309,903(\mathrm{p}=0,000)$ permitiram julgar adequada a aplicação da análise fatorial, pois, no caso do teste de esfericidade, o valor de $\mathrm{p}$ tende a zero e no teste $\mathrm{KMO}>0,5$.

Ambos indicam a adequação dos dados para a realização da análise fatorial. $\mathrm{O}$ teste Kaiser-Meyer-Olkin (KMO) é uma estatística que indica a proporção da variância dos dados que pode ser considerada comum a todas as variáveis, ou seja, que pode ser atribuída a um fator comum, então: quanto mais próximo de 1 (unidade) melhor o resultado, ou seja, mais adequada é a amostra à aplicação da análise fatorial.

O teste de esfericidade de Bartlett testa se a matriz de correlação é uma matriz identidade, o que indicaria que não há correlação entre os dados. Dessa forma, procura-se para um nível de significância assumido em 5\% rejeitar a hipótese nula de matriz de correlação identidade.

A fim de verificar a dimensionalidade do instrumento, foi realizada uma análise exploratória dos dados e análise dos componentes principais. Posteriormente, utilizou-se a técnica dos eixos principais com rotação VARIMAX. A fim de assegurar que cada item representasse o construto subjacente ao fator, foi estipulada uma carga fatorial mínima de 0,40 para aceitar o item.

A Análise Fatorial para componentes principais com rotação $V A R I M A X$ tem por objetivo aglutinar itens do instrumento que definem dimensões específicas. Essa etapa foi 
realizada de acordo com o critério de Kaiser (1960) para revelar fatores com autovalores maiores que 1; convém lembrar que a opção pela rotação ortogonal teve como objetivo minimizar a relação entre os fatores.

Reporta-se, também, o percentual de variância explicada pelo fator extraído para cada dimensão, bem como os autovalores associados ao fator único extraído, que sendo maiores que a unidade indicam que apenas um fator, de fato, seria relevante para extrair os componentes comuns aos dados.

A análise fatorial demonstrou a presença de quatro fatores com autovalores superiores a 1,4, os quais serão considerados neste estudo. O total de fatores explica $45,21 \%$ da variância total das respostas dos professores de Estatística. O primeiro fator explica 17,55\%, o segundo $10,13 \%$, o terceiro $9,61 \%$ e o quarto $7,92 \%$ (Tabela 1 ).

Tabela 1. Fatores obtidos pela análise fatorial, com valor próprio maior do que 1,00 , obtidos a partir da Análise dos Componentes Principais, relativamente aos itens da escala de avaliação das atitudes do professores de Estatística em relação à Estatística.

\begin{tabular}{lccc}
\hline \multicolumn{1}{c}{ Fator } & Valor próprio & Variância explicada (\%) & Variância acumulada (\%) \\
\hline Cognitivo & 3,159 & 17,55 & 17,55 \\
Educativo & 1,822 & 10,13 & 27,68 \\
Afetivo e social & 1,729 & 9,61 & 37,29 \\
Conduta & 1,426 & 7,92 & 45,21 \\
\hline
\end{tabular}

Além disso, foi utilizado o recurso gráfico do Scree Test, que, segundo Cattell (1966), ilustra como a variabilidade dos dados está distribuída entre os eixos da ordenação, permitindo identificar o número de eixos mais significativos, a qual apresenta a distribuição gráfica dos autovalores e componentes. Observa-se a possibilidade de realização da análise fatorial com quatro fatores (Figura 1).

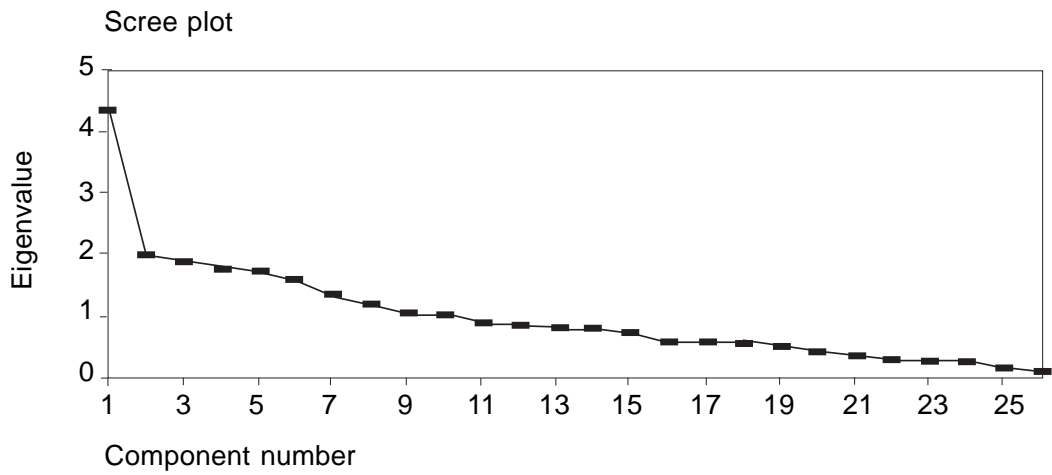

Figura 1. Gráfico de sedimentação. 
A Tabela 2 aponta as cargas fatoriais para cada item dos quatro fatores resultantes. Os sete itens que compõem o primeiro fator se referem ao componente cognitivo; o segundo fator é composto por quatro itens e refere-se ao componente educativo; o terceiro fator, composto por seis itens, refere-se ao componente afetivo e social; e, por fim, o quarto componente é composto por quatro itens referentes ao componente de conduta.

Tabela 2. Extração das cargas fatoriais dos itens que compõem a escala de avaliação das crenças de eficácia do professor, com rotação VARIMAX.

\begin{tabular}{|c|c|c|c|c|c|}
\hline $\begin{array}{l}\mathrm{N}^{\circ} \text { do } \\
\text { item }\end{array}$ & Itens & Fator 1 & Fator 2 & Fator 3 & Fator 4 \\
\hline 1 & $\begin{array}{l}\text { A aprendizagem da Estatística não pressupõe } \\
\text { conhecimentos matemáticos. }\end{array}$ & 0,592 & & & \\
\hline 2 & $\begin{array}{l}\text { É importante apresentar conceitos básicos da } \\
\text { Estatística Bayesiana. }\end{array}$ & 0,414 & & & \\
\hline 3 & $\begin{array}{l}\text { Vínculo a Estatística aos métodos e técnicas } \\
\text { científicas. }\end{array}$ & 0,505 & & & \\
\hline 4 & $\begin{array}{l}\text { Estudo e procuro explicações lógicas que comprovem } \\
\text { as imprecisões apresentadas por autores de livros de } \\
\text { Estatística. }\end{array}$ & 0,422 & & & \\
\hline 5 & $\begin{array}{l}\text { Procuro diferentes maneiras de resolver um problema } \\
\text { de Estatística. }\end{array}$ & 0,556 & & & \\
\hline 6 & $\begin{array}{l}\text { A Estatística ajuda a solucionar problemas } \\
\text { objetivamente. }\end{array}$ & 0,581 & & & \\
\hline 7 & $\begin{array}{l}\text { A Estatística me ajuda a entender mais profundamente } \\
\text { a complexidade de certos temas. }\end{array}$ & 0,678 & & & \\
\hline 8 & $\begin{array}{l}\text { É importante desenvolver pesquisas para que os } \\
\text { alunos possam fazer a relações entre a teoria e a } \\
\text { prática. }\end{array}$ & & 0,713 & & \\
\hline 9 & $\begin{array}{l}\text { Fazer perguntas aos alunos durante as aulas ajuda na } \\
\text { apreensão do conteúdo. }\end{array}$ & & 0,655 & & \\
\hline 10 & $\begin{array}{l}\text { Não me parece importante relacionar novos conceitos } \\
\text { com conteúdos anteriormente apreendidos. }\end{array}$ & & 0,469 & & \\
\hline 11 & $\begin{array}{l}\text { Devo desenvolver atividades com dados reais } \\
\text { utilizando minhas experiências. }\end{array}$ & & 0,720 & & \\
\hline 12 & É divertido lecionar estatística. & & & 0,470 & \\
\hline 13 & $\begin{array}{l}\text { Os alunos devem estar conscientes da importância do } \\
\text { conhecimento matemático para a aprendizagem da } \\
\text { Estatística. }\end{array}$ & & & 0,502 & \\
\hline 14 & $\begin{array}{l}\text { Motivar os alunos ajuda na aprendizagem da } \\
\text { Estatística. }\end{array}$ & & & 0,476 & \\
\hline 15 & $\begin{array}{l}\text { O pensamento estatístico é tão necessário para a } \\
\text { cidadania eficiente como saber ler e escrever. }\end{array}$ & & & 0,473 & \\
\hline 16 & $\begin{array}{l}\text { Podemos manipular a realidade por meio da } \\
\text { Estatística. }\end{array}$ & & & 0,494 & \\
\hline 17 & $\begin{array}{l}\text { Respondo com maturidade quando meus alunos } \\
\text { fazem uma pergunta. }\end{array}$ & & & 0,428 & \\
\hline 18 & $\begin{array}{l}\text { As representações gráficas não facilitam a } \\
\text { compreensão dos resultados estatísticos. }\end{array}$ & & & & 0,563 \\
\hline 19 & Evito as informações estatísticas quando as leio. & & & & 0,795 \\
\hline 20 & $\begin{array}{l}\text { Evito ler artigos científicos onde são apresentados } \\
\text { resultados estatísticos. }\end{array}$ & & & & 0,622 \\
\hline \multirow[t]{4}{*}{21} & Utilizo a Estatística exclusivamente para dar aulas. & & & & 0,559 \\
\hline & Número de itens & 7 & 4 & 6 & 4 \\
\hline & \% da variância & 17,55 & 10,13 & 9,61 & 7,92 \\
\hline & Alfa de Cronbach & 0,65 & 0,68 & 0,51 & 0,63 \\
\hline
\end{tabular}


A escala ficou composta por 21 itens e obteve um coeficiente alfa de Cronbach igual a 0,76 . Os fatores extraídos foram denominados cognitivo, educativo, afetivo/social e de conduta, com alfas de Cronbach de 0,65; 0,68; 0,51 e 0,63, respectivamente.

Os resultados descritivos demonstram as correlações existentes entre os quatro fatores (Tabela 3). O fator 2 (educativo), o fator 3 (afetivo e social ) e o fator 4 (de conduta) apresentam-se diretamente correlacionados entre si, ou seja, há uma relação positiva entre estes componentes. Os únicos fatores que não apresentam correlação significativa são o fator 1 (cognitivo) com o fator 4 (de conduta), indicando que estes fatores se apresentam independentes entre si, ou seja, os aspectos cognitivos das atitudes dos professores em relação à Estatística não interferem nos aspectos de conduta ou comportamental destes mesmos professores.

Tabela 3. Correlação entre os quatro fatores (Cognitivo, Educativo, Afetivo/social e de Conduta)

\begin{tabular}{lcccr}
\hline & $\begin{array}{c}\text { Fator 1 } \\
\text { Cognitivo }\end{array}$ & $\begin{array}{c}\text { Fator 2 } \\
\text { Educativo }\end{array}$ & $\begin{array}{c}\text { Fator 3 } \\
\text { Afetivo e social }\end{array}$ & $\begin{array}{r}\text { Fator 4 } \\
\text { Conduta }\end{array}$ \\
\hline Fator 1 Cognitivo & 1,000 & $0,233^{*}$ & 0,386 & 0,145 \\
Fator 2 Educativo & $0,233^{*}$ & 1,000 & 0,353 & $0,314^{\star *}$ \\
Fator 3 Afetivo e social & $0,386^{\star *}$ & $0,353^{\star *}$ & 1,000 & $0,278^{\star *}$ \\
Fator 4 Conduta & 0,145 & $0,314^{\star *}$ & $0,278^{* *}$ & 1,000 \\
\hline
\end{tabular}

* Correlação é significativa $(p<0,05)$

** Correlação é significativa $(p<0,01)$

\section{Discussão}

A interpretação dos fatores foi realizada partindo da análise dos itens que mais intensificam a consistência de cada fator (Tabela 2). Foi observada, também, a análise das correlações entre os fatores (Tabela 3).

No primeiro fator, o qual diz respeito ao componente cognitivo, percebemos que os itens de maior carga se referem ao professor acreditar que a Estatística o ajuda a entender mais profundamente a complexidade de temas diversos (item 7), e que a aprendizagem da Estatística pressupõe conhecimentos matemáticos (item 1, Tabela 2). Estes itens corroboram a definição desse componente que são expressões do pensamento. Estes atributos, que são associados com o objeto atitude, expressam avaliação positiva ou negativa, indo de posturas extremamente positivas a posturas extremamente negativas, como exemplo: alguns professores acreditam que os alunos não assimilam com facilidade conteúdos de probabilidade. Esta crença associa o objeto atitude com um atributo negativo. Outros professores podem acreditar que os conhecimentos matemáticos desenvolvidos no Ensino Médio são fundamentais para a aprendizagem da Probabilidade, ou seja, esta crença associa o objeto da atitude a um atributo positivo.

O segundo fator refere-se ao componente educativo (Tabela 2), é o interesse do professor em relação à Estatística e sua aprendizagem. Refere-se à relação com as atividades educativas, e que nem sempre o professor tem consciência desta sua prática. O conteúdo dos itens com o mais elevados níveis de carga neste fator são as posturas educacionais em que o profes- 
sor acredita ter que desenvolver atividades com dados reais utilizando suas experiências (item 11); e que considera ser importante desenvolver pesquisas para que os alunos possam fazer as relações entre a teoria e a prática (item 8). É papel do professor promover práticas pedagógicas que encorajem os alunos a pensarem criticamente e promoverem a capacidade destes para avaliarem criticamente a informação numa sociedade cada vez mais baseada na mídia.

O terceiro fator refere-se ao componente afetivo e social (Tabela 2). Ele se refere às expressões do sentimento do professor no processo ensino-aprendizagem e à valorização do papel da Estatística no âmbito sociocultural. Os itens de maior carga referem-se: ao professor ter a consciência de que é necessário passar ao seu aluno a importância do conhecimento matemático para a aprendizagem da Estatística (item 13); que podemos manipular a realidade por meio da Estatística (item 16), e que o pensamento estatístico é tão necessário para a cidadania eficiente como saber ler e escrever (item 15, Tabela 2). As respostas do tipo afetivo consistem de sentimentos, humor, emoções e atividades do sistema nervoso simpático que as pessoas experimentam em relação ao objeto da atitude. Acredito na ideia de que nós, professores, devemos ser amantes de nossa profissão, comprometidos com a produção do conhecimento em sala de aula, que desenvolvem com seus alunos um vínculo muito estreito de amizade e respeito mútuo pelo saber. Professores, ou melhor, educadores que não medem esforços para levar seus alunos à ação, à reflexão crítica, à curiosidade, ao questionamento e à descoberta são essenciais.

O quarto e último fator é referente ao componente de conduta. Destacando os itens 19 e 20, pode-se considerar a existência, nesses itens, da necessidade de se buscarem informações estatísticas na mídia em geral e na leitura de artigos científicos. Já o item com menor carga alude ao professor que expressa sua posição quanto a utilizar a Estatística exclusivamente para dar aulas (item 21, Tabela 2). As respostas do tipo comportamental (ou de conduta) consistem da ação que as pessoas exibem em relação ao objeto da atitude. Tanto quanto as respostas cognitivas, educativas, afetivas e sociais podem também variar de atitudes extremamente positivas a extremamente negativas. Assim, se alguns professores de Estatística não utilizam softwares estatísticos em suas aulas, por acreditarem que não facilitam a aprendizagem dos conteúdos ministrados, outros aplicam instrumentos para estabelecerem perfil dos alunos, acreditando que um trabalho prático auxilia infinitamente na apreensão dos mesmos conteúdos. Geralmente, pessoas que assumem atitudes favoráveis fomentam ou apoiam seu comportamento, e pessoas que assumem atitudes desfavoráveis tendem a engajar-se em comportamentos que retardem ou estejam em oposição ao fato.

No tocante à análise correlacional dos fatores, foi possível perceber que o fator 4 (componente de conduta do professor) foi o único que não apresentou-se correlacionado com todos os fatores considerados, indicando autonomia em relação ao componente cognitivo.

\section{Considerações finais}

A análise fatorial da Escala de Atitudes dos Professores de Estatística em relação à Estatística (EAPE) possibilitou a identificação dos quatro fatores existentes no instrumento, os quais explicam $44,21 \%$ da variância total encontrada. O primeiro fator, referente ao componente cognitivo, explica $17,55 \%$ da variância; já o segundo, o qual diz respeito ao compo- 
nente educativo, explica 10,13\%; o terceiro, que diz respeito ao componente afetivo e social, explica 9,61\%; e o quarto e último, que faz menção ao componente da conduta, explica 7,92\% da variância. Sendo assim, foi possível verificar que o instrumento tem uma estrutura fatorial consistente.

A análise desenvolvida neste estudo salienta a importância de pesquisas relativas a se conhecer mais sobre o universo de professores de Estatística no Brasil, já que nos deparamos, na literatura vigente, com raros estudos concernentes a este grupo, que cada vez mais tem reconhecida sua atuação na formação de profissionais, tanto no setor comercial quanto no setor acadêmico. Assim, o presente trabalho encontra, também, sua relevância na amostra escolhida. Ao mesmo tempo, os resultados alcançados sugerem a necessidade de novas investigações que possibilitem validar o instrumento em amostras diversificadas para os professores de Estatística. Da mesma forma, salienta-se a necessidade da elaboração de estudos em profundidade no que concerne às diversas estratégias educacionais utilizadas por este grupo.

De acordo com a nossa experiência docente, acreditamos que o conhecimento deveria ser construído pelos estudantes à medida que as atividades didáticas fizessem uso de significados lógicos, de ideias relevantes, como, também, de ideias que já se encontram disponíveis na estrutura cognitiva deste. Para que o professor consiga levar seus estudantes a uma aprendizagem significativa, possibilitando a formação de conceitos e habilidades para solucionar problemas, faz-se necessário colocar em prática uma das ações fundamentais do ensino, que é a de trabalhar os conteúdos na perspectiva da sua relevância, viabilidade, utilidade e significado. A partir disto, os conteúdos escolares se relacionariam com a prática e cotidiano dos alunos, possibilitando aos mesmos atribuírem sentido ao que lhes é ensinado.

\section{Referências}

APARICIO, A; BAZÁN, J; ABDOUNUR, O. Atitude e desempenho em relação à Estatística em professores de Ensino Fundamental no Peru: primeiros resultados. In: ENCONTRO PAULISTA DE EDUCAÇÃO MATEMÁTICA (EPEM), 7., 2004, São Paulo. Anais ... São Paulo, 2004. Disponível em: <http://www.sbempaulista.org.br/epem/ anais/Comunicacoes_Orais/co0009.doc>. Acesso em: 21 set. 2009.

AUZMENDI, E. Las actitudes hacia la matemática estadística en las enseñanzas medias y universitarias. Bilbao: Mensajero, 1992.

BRITO, M. R. F. Adaptação e validação de uma Escala de Atitudes em relação à Matemática. Zetetiké, Campinas, v. 6, n. 9, p. 109-162, 1998.

CATTELL, R. B. The scree test for the number of factors. Multivariate Behavioral Research, Norman, v. 1, n. 2, p. 245-276, 1966. 
Validação da escala de atitude de professores ...

CAZORLA, I. M. et al. Adaptação e validação de uma Escala de Atitudes em relação à

Estatística. In: CONFERÊNCIA INTERNACIONAL EXPERIÊNCIAS E

EXPECTATIVAS DO ENSINO DE ESTATÍSTICA - DESAFIOS PARA O SÉCULO

XXI, 1999, Florianópolis. Atas... Santa Catarina: Florianópolis, 1999. p. 45-57.

ESTRADA, A.; BATANERO, C.; FORTUNY, J. M. Actitudes y estadistica en profesores en formación y en ejercicio. In: CONGRESO NACIONAL DE ESTADÍSTICA E INVESTIGACIÓN OPERATIV, 27., 2003, Lleida. Actas... Lleida: Edicions de la Universitat de Lleida, 2003. p. 909-920.

GIL FLORES, J. Actitudes hacia la Estadística: incidencia de las variables sexo y formación previa. Revista Española de Pedagogía, Madrid, v. LVII, n. 214, p. 567-590, 1999.

GÓMEZ CHACÓN, I. M. Matemática emocional: os afetos na aprendizagem matemática. Porto Alegre: Artmed, 2003.

KAISER, H. F. The application of electronic computers to factor analysis. Educational and Psychological Measurement, Thousand Oaks, v. 20, p. 141-151, 1960.

MacNAB, D. S.; PAYNE, F. Beliefs, attitudes and practices in Mathematics teaching: perceptions of Scottish primary school student teachers. Journal of Education for Teaching, Manchester, v. 29, n. 1, p. 55-68, 2003.

NASSER, F. Prediction of statistics achievement. In: INTERNATIONAL STATISTICAL INSTITUTE, 52., 1999, Helsinki. Proceedings... Helsinki, Finland, 1999. v. 3, p $7-8$.

Structural model of the effects of cognitive and affective factors on the achievement of arabic-speaking pre-service teachers in introductory statistics. Journal of Statistics Education, Alendalle, v. 12, n. 1, 2004. Disponível em: <http://www.amstat.org/ publications/jse/v12n1/nasser.html>. Acesso em: 21 set. 2009.

ONWUEGBUZIE, A. J. The dimensions of statistics anxiety: a comparison of prevalence rates among mid-southern university students. Louisiana Educational Research Journal, Louisiana, v. 23, n. 2, p. 23-40, 1998.

. Expanding the framework of internal and external validity in quantitative research.

Research in the Schools, v. 10, n. 1, p. 71-90, 2003.

WISENBAKER, J.; NASSER, F. A.; SCOTT, J. A cross-cultural comparison of path models relating attitudes about and achievement in introductory statistics courses. In: INTERNATIONAL STATISTICAL INSTITUTE, 52., 1999, Helsinki. Proceedings... Helsinki, Finland, 1999. Disponível em: < http://www.stat.fi/isi99/proceedings/arkisto/ varasto/wise0263.pdf>. Acesso em: 21 set. 2009.

Artigo recebido em abril de 2009 e aceito em agosto de 2009. 
